\title{
The Trotter-Kato Product Formula for Gibbs Semigroups
}

\author{
Hagen Neidhardt* and Valentin A. Zagrebnov \\ Laboratory of Theoretical Physics, Joint Institute for Nuclear Research, \\ SU-141980 Dubna, USSR
}

\begin{abstract}
The trace-norm convergence of the Trotter-Lie product formula has recently been proved for particular classes of Gibbs semigroups. In the present paper we prove it for the whole generality including generalization of the product formula proposed by Kato.
\end{abstract}

\section{Introduction}

Let $A$ and $B$ be linear operators in a separable complex Hilbert space $\mathscr{H}$. Then, under suitable conditions concerning $\{A, B\}$ the strong limit

$$
s-\lim _{n \rightarrow \infty}\left(\exp \left(-\frac{t}{n} A\right) \exp \left(-\frac{t}{n} B\right)\right)^{n}=\exp (-t C)
$$

exists for $t \geqq 0$, where the operator $C$ can be constructed by means of $A$ and $B$. This is the well-known Trotter-Lie product formula for strongly continuous $\left(C_{0^{-}}\right)$ semigroups [1]. (For finite matrices it has been established by Sophus Lie about 1875.) Since the discovery of the product formula, it has permeated through mathematics and mathematical physics, challenging the problem of relaxation and generalization of the hypotheses under which the formula holds, see [2-10].

A solution of this problem implies that one has to do the following:

(i) to find the set of pairs $\{A, B\}$ for which the limit (1.1) exists;

(ii) to identify the operator $C$ and to describe the mapping $\{A, B\}: \rightarrow C$;

(iii) to generalize (if possible) the exponential functions involved in the left-hand side of (1.1) to a class of real-valued, Borel measurable functions $f(\cdot), g(\cdot)$ such that in some operator topology $\tau$,

$$
\tau-\lim _{n \rightarrow \infty}\left(f\left(\frac{t}{n} A\right) g\left(\frac{t}{n} B\right)\right)^{n}=\exp (-t C) \Pi
$$

* On leave of absence from Karl-Weierstrass-Institut für Mathematik, Adw der DDR, Mohrenstrasse 39, DDR-1086 Berlin 
for $t \in \mathbb{R}_{+}^{1}=\left\{x \in \mathbb{R}^{1}: x \geqq 0\right\}$ (or its continuation into the right complex half-plane) where $\Pi$ is the orthogonal projection from $\mathscr{H}$ onto the closed subspace in which the operator $C$ is defined;

(iv) to indicate a natural topology $\tau$ in which the convergence in (1.2) will take place.

A lot of papers has been devoted to points (i) and (ii) of the above program when $\{A, B\}$ is a pair of self-adjoint operators. It was Trotter [1] who for the first time proved (1.1) for $C_{0}$-semigroups whenever the operators $A$ and $B$ are semibounded from below and the algebraic sum $A+B$ is essentially self-adjoint on a common dense domain $\mathscr{D}=\mathscr{D}(A) \cap \mathscr{D}(B)$, i.e., the operator $A+B$ has a unique self-adjoint extension defined by the closure $(A+B)^{-}=C$. For unitary groups $(t \rightarrow i t)$, the semiboundedness can be cancelled. The proviso about the domain $\mathscr{D}$ is important because there exist examples of non-negative self-adjoint operators $\{A, B\}$ such that $\mathscr{D}(A) \cap \mathscr{D}(B)=\{0\}$ and $\left(\mathscr{D}\left(A^{1 / 2}\right) \cap \mathscr{D}\left(B^{1 / 2}\right)\right)^{-}=\mathscr{H}$. Therefore, Chernoff [2] (see also Faris [3] and Simon [4]) has extensively studied (1.1) for $C_{0^{-}}$ contraction semigroups to define a generalized sum of two unbounded nonnegative self-adjoint operators $A$ and $B$ whenever their common form domain $Q=\mathscr{D}\left(A^{1 / 2}\right) \cap \mathscr{D}\left(B^{1 / 2}\right)$ is dense in $\mathscr{H}$ and the quadratic form $t_{B}[u]=\left\|B^{1 / 2} u\right\|^{2}$, $u \in Q(B)=\mathscr{D}\left(B^{1 / 2}\right)$ is bounded relative to $t_{A}[u]: Q(A) \subset Q(B)$ and $t_{B}[u] \leqq a\|u\|^{2}$ $+b t_{A}[u], u \in Q(A)$, for some $a, b \geqq 0$. Then, $C=A+B$ is the form sum of $A$ and $B$ [5], i.e. a unique non-negative self-adjoint operator associated with the nonnegative closed quadratic form

$$
h[u]=t_{A}[u]+t_{B}[u], u \in Q(A) \cap Q(B) .
$$

The essential contribution to the theory at point (iii) has been made by Kato $[6,7]$. In two subsequent papers he has proved the product formula (1.2) in the strong operator topology $\tau=s$ for a very general (but a natural) class of real-valued functions $f, g: \mathbb{R}_{+}^{1} \rightarrow[0,1]$ and an arbitrary pair $\{A, B\}$ of non-negative unbounded self-adjoint operators in the Hilbert space $\mathscr{H}$. Then, $\Pi$ is the orthogonal projection from $\mathscr{H}$ onto the closed subspace $\mathscr{H}^{\prime}$ spanned by $Q=\mathscr{D}\left(A^{1 / 2}\right) \cap \mathscr{D}\left(B^{1 / 2}\right)$ and $C=A \dot{+} B$ is the self-adjoint operator in $\mathscr{H}^{\prime}$ associated with the non-negative closed quadratic form $h[u]=\left\|A^{1 / 2} u\right\|^{2}+\left\|B^{1 / 2} u\right\|^{2}, u \in Q$, which is densely defined in $\mathscr{H}^{\prime}$. In the first paper [6] he has proved the product formula (1.2) for pairs of functions $\{f(x), g(x)\}$ including $\left\{e^{-x},(1+x)^{-1}\right\},\left\{(1+x)^{-1}, e^{-x}\right\}$ and $\left\{(1+x)^{-1}\right.$, $\left.(1+x)^{-1}\right\}$, while in the second one [7] a completely different proof, which allows one to include the important case of $\left\{e^{-x}, e^{-x}\right\}$, has been proposed. Below we shall refer to (1.2) for $\tau=s$ as the Trotter-Kato product formula.

The problem to prove the Trotter-Kato product formula for unitary groups and imaginary resolvents $\{f, g\}=\left\{(1+i x)^{-1},(1+i x)^{-1}\right\}$ has been considered by Lapidus $[8,9]$.

Recently, one of the authors of the present paper has made an attempt to connect the topology $\tau$ in the product formula with that in which semigroups involved in (1.1) are continuous for $t \in \mathbb{R}_{+}^{1} \backslash\{0\}$ [10]. This question has been inspired by point (iv) of the above program for Gibbs semigroups, see [11, 12]. If at least one of the operators $A$ or $B$ generates a self-adjoint Gibbs semigroup and Trotter's conditions on the pair $\{A, B\}$ are satisfied, then the strong operator convergence in (1.1) can be lifted for $t>0$ to $\tau=\|\cdot\|_{1}$-topology (trace-norm convergence). 
In this paper, we continue these investigations related to (iv). We prove the Trotter-Kato product formula (1.2) for $\tau=\|\cdot\|_{1}$ when at least one operator of the pair $\{A, B\}$ is a generator of a self-adjoint Gibbs semigroup. Furthermore, relaxation of the conditions on $\{A, B\}$ imposed by Kato $[6,7]$, which are relevant in applications to quantum statistical mechanics, is discussed.

To formulate the problem more precisely we recall some notation and definitions, see e.g. [13]. If $\mathscr{H}$ is a separable Hilbert space, then $\mathscr{C}_{p}(\mathscr{H})$ is the Banach space of compact operators on $\mathscr{H}$ with finite $\|\cdot\|_{p}$-norm:

$$
\|X\|_{p}=\left\{\sum_{k=1}^{\infty}\left(\lambda_{k}(X)\right)^{p}\right\}^{1 / p}, \quad 1 \leqq p<\infty .
$$

Here $\left\{\lambda_{k}(X)\right\}_{k=1}^{\infty}$ are the singular values of the operator $X \in \mathscr{C}_{p}(\mathscr{H})$, i.e. eigenvalues of the operator $|X|=\left(X^{*} X\right)^{1 / 2}$. The Banach spaces $\left\{\mathscr{C}_{p}(\mathscr{H})\right\}_{1 \leqq p<\infty}$ are $*$-ideals in the Banach space of compact operators $\operatorname{Com}(\mathscr{H})=\mathscr{C}_{\infty}(\mathscr{H})$ and bounded operators $\mathscr{B}(\mathscr{H})$ in $\mathscr{H}$ ordered by

$$
\mathscr{C}_{1}(\mathscr{H}) \subset \mathscr{C}_{2}(\mathscr{H}) \subset \ldots \subset \mathscr{C}_{p}(\mathscr{H}) \subset \ldots \subset \operatorname{Com}(\mathscr{H}) \subset \mathscr{B}(\mathscr{H}) .
$$

Definition 1.1 [11]. A $C_{0}$-semigroup $\{G(t)\}_{t \geqq 0}$ in a separable Hilbert space $\mathscr{H}$ is called a Gibbs semigroup if $G(t):(0, \infty) \rightarrow \mathscr{C}_{1}(\mathscr{H})$.

Remark 1.2. From the continuity of multiplication (Grümm [14]):

$$
X_{n} Y_{n} \stackrel{\|\cdot\|_{p}}{\longrightarrow} X Y \quad \text { if } \quad X_{n} \stackrel{s}{\longrightarrow} X, Y_{n} \stackrel{\|\cdot\|_{P}}{\longrightarrow} Y \text { as } n \rightarrow \infty
$$

for $\left\{X_{n}\right\}_{n \geqq 1} \in \mathscr{B}(\mathscr{H}),\left\{Y_{n}\right\}_{n \geqq 1} \in \mathscr{C}_{p}(\mathscr{H}), 1 \leqq p<\infty$, it follows that the Gibbs semigroups are $\|\cdot\|_{1}$-continuous for $t>0$.

The Gibbs semigroups naturally arise in quantum statistical mechanics (QSM) as one-parameter self-adjoint $C_{0}$-semigroups generated by a Hamiltonian $H: G_{H}(\beta)=\exp (-\beta H)$. Here, a parameter $\beta>0$ is nothing but the inverse temperature of the system described by the operator $H$. For continuous systems of QSM, $H$ is a sum of two parts: an ideal (kinetic-energy operator $T$ ) and a nonideal (interaction operator $U$ ). It is known [4] that for singular two-body potentials the operator $U$ is not small with respect to the kinetic-energy operator $T$ in the usual operator sense [5]. Therefore, in this case the definition of the Hamiltonian of the system is not very obvious. Moreover, as far as in the QSM the main object of investigations is the partition function $Z(\beta)=\operatorname{Tr}\left(G_{H}(\beta)\right)$, regularizations or limit procedures defining the Hamilitonian $H$ have to be such that the corresponding families of operators approximating the Gibbs semigroup $G_{H}(\beta)$ should be $\|\cdot\|_{1^{-}}$ convergent $[11,15]$. The same arguments are applied to the Trotter product formula which is often used (under the Tr) for constructing a sum of $T$ and $U$, trace Feynman-Kac formula and other calculations, see e.g. [16].

The outline of the paper is as follows. In Sect. 2 we accumulate technical preliminaries which in our opinion have also their own interest for the theory of the *-ideals $\mathscr{C}_{p}(\mathscr{H}), 1 \leqq p \leqq \infty$. In Sect. 3 , we prove the Trotter-Kato product formula (1.2) in $\|\cdot\|_{1}$-topology. This is done in two steps. First, we consider a special case when conditions similar to [6] plus the requirement that $f(t A) \in \mathscr{C}_{p}(\mathscr{H})$ for $t>0$ and $1 \leqq p<\infty$ are fulfilled. In contrast with the case of $C_{0}$-semigroups and $\tau=s$ (see 
[7]) we cannot avoid this intermediate step exploiting monotony properties of auxiliary operator families. The vindication of this line of reasoning becomes clear when one follows the proof of the product formula (1.2) in the general case of conditions à la [7]. The last Sect. 4 is devoted to concluding remarks and possible applications.

\section{Technical Preliminaries}

In the following, we prove some kind of bounded convergence theorem for trace ideals which will be necessary in the sequel.

For $K, L \in \mathscr{B}(\mathscr{H})$ we write $K \ll L$ if $\|K u\| \leqq\|L u\|, u \in \mathscr{H}$. This condition is equivalent to $K^{*} K \leqq L^{*} L$ and also to $|K| \ll|L|$. It is obvious that $K \ll L$ with $L \in \mathscr{C}_{p}(\mathscr{H})$ implies that $K \in \mathscr{C}_{p}(\mathscr{H})$ and $\|K\|_{p} \leqq\|L\|_{p}, 1 \leqq p<+\infty$.

Proposition 2.1. Let $K_{n}=K_{n}^{*} \in \mathscr{B}(\mathscr{H}), J_{n}=J_{n}^{*} \in \mathscr{B}(\mathscr{H}), n=1,2, \ldots$. Assume that $s-\lim _{n \rightarrow \infty} J_{n}=I$ and that $K_{n} \ll L J_{n}, n=1,2, \ldots$, where $L=L^{*} \in \mathscr{C}_{p}(\mathscr{H})$ for some $1 \leqq p<+\infty$ (so that $K_{n} \in \mathscr{C}_{p}(\mathscr{H})$ ). If $w$ - $\lim _{n \rightarrow \infty} K_{n}=K$, then $K \in \mathscr{C}_{p}(\mathscr{H})$ and \|\|$_{p}-\lim _{n \rightarrow \infty} K_{n}=K$.

Proof. By $w$ - $\lim _{n \rightarrow \infty} K_{n}=K$ we find $\|K u\| \leqq \lim _{n \rightarrow \infty} \inf \left\|K_{n} u\right\| \leqq \lim _{n \rightarrow \infty} \inf \left\|L J_{n} u\right\|$ $=\|L u\|, u \in \mathscr{H}$. Hence $K \ll L$ and $K \in \mathscr{C}_{p}(\mathscr{H})$.

Since $L \in \mathscr{C}_{p}(\mathscr{H})$ there is a sequence $\left\{P_{j}\right\}_{j=1}^{\infty}$ of finite-dimensional orthogonal projections such that $L P_{j}=P_{j} L, j=1,2, \ldots$, and $\left\|L Q_{j}\right\|_{p}=\left\|Q_{j} L\right\|_{p}<\frac{1}{j}, j=1,2, \ldots$, where $Q_{j}=I-P_{j}$. By $\quad w-\lim _{n \rightarrow \infty} K_{n}=K$ we have $\|\cdot\|_{p}-\lim _{n \rightarrow \infty} P_{j} K_{n} P_{j}=P_{j} K P_{j}$ for every $j=1,2, \ldots$, and every $1 \leqq p<+\infty$. Given $\varepsilon>0$, therefore, we have $\left\|P_{j}\left(K_{n}-K\right) P_{j}\right\|_{p}<\varepsilon$ for sufficiently large $n$ depending on $\varepsilon$ and $j$. On the other hand, we have $\left\|\left(K_{n}-K\right) Q_{j}\right\|_{p} \leqq\left\|K_{n} Q_{j}\right\|_{p}+\left\|K Q_{j}\right\|_{p} \leqq\left\|Q_{j} L J_{n} Q_{j}\right\|_{p}+\left\|L P_{j} J_{n} Q_{j}\right\|_{p}$ $+\left\|L Q_{j}\right\|_{p}$. Since $s-\lim _{n \rightarrow \infty} J_{n}=I$, we have $\sup _{n}\left\|J_{n}\right\|=c<+\infty$. Consequently, we obtain the estimate

$$
\left\|\left(K_{n}-K\right) Q_{j}\right\|_{p} \leqq \frac{1+c}{j}+\|L\|\left\|Q_{j} J_{n} P_{j}\right\|_{p},
$$

$j=1,2, \ldots$ Since $P_{j}$ is a finite-dimensional projection, we get $\|\cdot\|_{p}-\lim _{n \rightarrow \infty} Q_{j} J_{n} P_{j}=0,1 \leqq p<+\infty$. Therefore, choosing $j$ and $n$ sufficiently large, we can achieve that $\left\|K_{n}-K\right\|_{p}<\varepsilon$. This proves the proposition.

Corollary 2.2. Let $K_{n}(\cdot): \mathscr{K} \rightarrow \mathscr{B}(\mathscr{H}), J_{n}(\cdot): \mathscr{K} \rightarrow \mathscr{B}(\mathscr{H}), n=1,2, \ldots$, and $K(\cdot): \mathscr{K}$ $\rightarrow \mathscr{B}(\cdot)$ operator-valued functions such that the assumptions of Proposition 2.1 are pointwise satisfied. If in addition $\left\|J_{n}(t)\right\| \leqq c<+\infty, \quad s-\lim _{n \rightarrow \infty} J_{n}(t)=I$ and $w$ - $\lim _{n \rightarrow \infty} K_{n}(t)=K(t)$ hold uniformly in $t \in \mathscr{K}$, then $\|\cdot\|_{p^{-}} \lim _{n \rightarrow \infty} K_{n}(t)=K(t)$ uniformly in $t \in \mathscr{K}$.

Proof. Since $w-\lim _{n \rightarrow \infty} K_{n}(t)=K(t)$ uniformly in $t \in \mathscr{K}$, we obtain that $\|\cdot\|_{p}-\lim _{n \rightarrow \infty} P_{j} K_{n}(t) P_{j}=P_{j} K(t) P_{j}$ uniformly in $t \in \mathscr{K}$. Further, replacing $K_{n}, K$ and 
$J_{n}$ by $K_{n}(t), K(t)$ and $J_{n}(t)$, respectively, the estimate (2.1) remains true. But the uniformity of $s-\lim J_{n}(t)=I$ in $t \in \mathscr{K}$ implies the uniformity of $\|\cdot\|_{p}-\lim _{n \rightarrow \infty} Q_{j} J_{n}(t) P_{j}=0$ in $t \in \mathscr{K}$, which completes the proof.

Proposition 2.3. Let $K_{n} \in \mathscr{C}_{2 p}(\mathscr{H})$ and $\hat{K}_{n} \in \mathscr{C}_{2 p}(\mathscr{H}), n=1,2, \ldots$, for some $p \in[1, \infty)$. Assume $s-\lim _{n \rightarrow \infty} K_{n}=K \in \mathscr{C}_{2 p}(\mathscr{H})$ and $\|\cdot\|_{2 p}-\lim _{n \rightarrow \infty} \hat{K}_{n}=K$. If $\left\|K_{n}\right\|_{2 p} \leqq\left\|\hat{K}_{n}\right\|_{2 p}$, $n=1,2, \ldots$, then $\|\cdot\|_{2 p^{-}} \lim _{n \rightarrow \infty} K_{n}=K$.

Proof. The proof is based on the inequality

$$
\|X+Y\|_{2 p}^{2 p}+\|X-Y\|_{2 p}^{2 p} \leqq 2^{2 p-1}\left\{\|X\|_{2 p}^{2 p}+\|Y\|_{2 p}^{2 p}\right\},
$$

$X, Y \in \mathscr{C}_{2 p}(\mathscr{H}), 1 \leqq p<+\infty$, which yields the uniform convexity of the Banach spaces $\mathscr{C}_{2 p}(\mathscr{H}), 1 \leqq p<+\infty$. Since $\left\|K_{n}\right\|_{2 p} \leqq\left\|\hat{K}_{n}\right\|_{2 p}, n=1,2, \ldots$, the sequence $\left\{\left\|K_{n}\right\|_{2 p}\right\}_{n=1}^{\infty}$ is uniformly bounded. Using $s$ - $\lim _{n \rightarrow \infty} K_{n}=K$ we find $w$ - $\|\cdot\|_{2 p}-\lim _{n \rightarrow \infty} K_{n}=K$, i.e. the sequence $\left\{K_{n}\right\}_{n=1}^{\infty}$ tends in the weak sense of the Banach space $\mathscr{C}_{2 p}(\mathscr{H})$ to $K$ as $n \rightarrow \infty$. But this implies $\|K\|_{2 p} \leqq \lim _{n \rightarrow \infty} \inf \left\|K_{n}\right\|_{2 p}$ $\leqq \lim _{n \rightarrow \infty} \sup \left\|K_{n}\right\|_{2 p} \leqq \lim _{n \rightarrow \infty}\left\|\hat{K}_{n}\right\|_{2 p}=\|K\|_{2 p}$. Hence, we find $\lim _{n \rightarrow \infty}\left\|K_{n}\right\|_{2 p}^{n \rightarrow \infty}=\|K\|_{2 p}$. In the same way, we get $\lim _{n \rightarrow \infty}\left\|K_{n}+K\right\|_{2 p}=2\|K\|_{2 p}$. Setting $X=K_{n}$ and $Y=K$ and taking into account (2.2) we complete the proof.

Corollary 2.4. Let $K_{n}(\cdot): \mathscr{K} \rightarrow \mathscr{C}_{2 p}(\mathscr{H}), \quad \hat{K}_{n}(\cdot): \mathscr{K} \rightarrow \mathscr{C}_{2 p}(\mathscr{H}), \quad n=1,2, \ldots, \quad$ and $K(\cdot): \mathscr{K} \rightarrow \mathscr{C}_{2 p}(\mathscr{H}), 1 \leqq p<+\infty$, be operator-valued functions such that the assumptions of Proposition 2.3 are pointwise satisfied. If in addition $s-\lim _{n \rightarrow \infty} K_{n}(t)=K(t)$, $\|\cdot\|_{2 p}-\lim _{n \rightarrow \infty} \hat{K}_{n}(t)=K(t)$ uniformly in $t \in \mathscr{K}$ and there is an $L \in \mathscr{C}_{2 p}^{n \rightarrow \infty}(\mathscr{H})$ such that $K(t) \ll L, t \in \mathscr{K}$, then $\|\cdot\|_{2 p^{-}} \lim _{n \rightarrow \infty} K_{n}(t)=K(t)$ uniformly in $t \in \mathscr{K}$.

Proof. Let us in accordance with the previous considerations introduce a family $\left\{P_{j}\right\}_{j=1}^{\infty}$ of finite-dimensional projections such that $L P_{j}=P_{j} L$ and $\left\|L Q_{j}\right\|_{2 p}<\frac{1}{j}$, $Q_{j}=I-P_{j}, j=1,2, \ldots$

Now we are going to show that $\lim _{n \rightarrow \infty}\left\|K_{n}(t)\right\|_{2 p}=\|K(t)\|_{2 p}$ uniformly in $t \in \mathscr{K}$. From the obvious inequality

$$
\left\|K_{n}(t) P_{j}\right\|_{2 p} \leqq\left\|K_{n}(t)\right\|_{2 p} \leqq\|\hat{K}(t)\|_{2 p},
$$

$n=1,2, \ldots, t \in \mathscr{K}$, we get the estimate

$$
\begin{gathered}
\left|\left\|K_{n}(t)\right\|_{2 p}-\|K(t)\|_{2 p}\right| \leqq\left\|K(t) Q_{j}\right\|_{2 p}+\|\left(K_{n}(t)\right. \\
-K(t)) P_{j}\left\|_{2 p}+\right\| \hat{K}_{n}(t)-K(t) \|_{2 p} .
\end{gathered}
$$


Since $K(t) \ll L$ we find that $\left\|K(t) Q_{j}\right\|_{2 p} \leqq\left\|L Q_{j}\right\|_{2 p}<\frac{1}{j}, j=1,2, \ldots$. Hence, we have

$$
\left|\left\|K_{n}(t)\right\|_{2 p}-\|K(t)\|_{2 p}\right|<\frac{1}{j}+\left\|\left(K_{n}(t)-K(t)\right) P_{j}\right\|_{2 p}+\left\|\hat{K}_{n}(t)-K(t)\right\|_{2 p},
$$

$n=1,2, \ldots, t \in \mathscr{K}$. Given $\varepsilon>0$ we fix a $j$ such that $\frac{1}{j}<\frac{\varepsilon}{3}$. Since $P_{j}$ is a finitedimensional projection we find that $\|\cdot\|_{2 p}-\lim _{n \rightarrow \infty} K_{n}(t) P_{j}=K(t) P_{j}$ uniformly in $t \in \mathscr{K}$. Consequently, there is a $n_{0}$ such that for $n \geqq n_{0}$ we have $\|\left(K_{n}(t)\right.$ $-K(t)) P_{j} \|_{2 p}<\frac{\varepsilon}{3}$ and $\left\|\hat{K}_{n}(t)-K(t)\right\|_{2 p}<\frac{\varepsilon}{3}$ uniformly in $t \in \mathscr{K}$. The last assertion follows by assumptions. Summing up we find $\left\|K_{n}(t)\right\|_{2 p} \underset{n \rightarrow \infty}{\longrightarrow}\|K(t)\|_{2 p}$ uniformly in $t \in \mathscr{K}$.

Similarly we prove that $\lim _{n \rightarrow \infty}\left\|K_{n}(t)+K(t)\right\|_{2 p}=2\|K(t)\|_{2 p}$ uniformly in $t \in \mathscr{K}$.

Since $\lim _{n \rightarrow \infty}\left\|K_{n}(t)\right\|_{2 p}=\|K(t)\|_{2 p}$ and $\|K(t)\|_{2 p} \leqq\|L\|_{2 p}$ uniformly in $t \in \mathscr{K}$, there is a $n_{0} \geqq 1$ such that the sequences $\left\{\left\|K_{n}(t)\right\|_{2 p}\right\}_{n=n_{0}}^{\infty}$ and $\left\{\left\|K_{n}(t)+K(t)\right\|_{2 p}\right\}_{n=n_{0}}^{\infty}$ are uniformly bounded in $t \in \mathscr{K}$. Hence, it is not hard to see that $\left\|K_{n}(t)\right\|_{2 p}^{2 p} \underset{n \rightarrow \infty}{\longrightarrow}\|K(t)\|_{2 p}^{2 p}$ and $\left\|K_{n}(t)+K(t)\right\|_{2 p}^{2 p} \underset{n \rightarrow \infty}{\longrightarrow} 2^{2 p}\|K(t)\|_{2 p}^{2 p}$ uniformly in

$t \in \mathscr{K}$. Taking into account (2.2) we get $\left\|K_{n}(t)-K(t)\right\|_{2 p}^{2 p} \underset{n \rightarrow \infty}{\longrightarrow} 0$ uniformly in $t \in \mathscr{K}$ which immediately completes the proof.

\section{Product Formula}

Let $A \geqq 0$ and $B \geqq 0$ be self-adjoint operators in a separable Hilbert space $\mathscr{H}$. Denoting by $Q=\mathscr{D}\left(A^{1 / 2}\right) \cap \mathscr{D}\left(B^{1 / 2}\right)$ we do not assume that $Q$ is dense in $\mathscr{H}$. By $\mathscr{H}^{\prime}$ we denote the closure of $Q$, i.e. $\mathscr{H}^{\prime}=Q^{-}$. In general, $\mathscr{H}^{\prime}$ is a proper subspace of $\mathscr{H}$, i.e. $\mathscr{H} \neq \mathscr{H}^{\prime}$. The orthogonal projection from $\mathscr{H}$ onto $\mathscr{H}^{\prime}$ is indicated by $\Pi$. We recall that $C$ is the self-adjoint operator in $\mathscr{H}^{\prime}$ associated with the non-negative closed quadratic form $f \rightarrow\left\|A^{1 / 2} f\right\|^{2}+\left\|B^{1 / 2} f\right\|^{2}, f \in Q$, i.e. $C=A \dot{+} B$.

Further, we introduce a class of Borel functions $f$ and $g$ defined on $\mathbb{R}_{+}^{1}=\left\{t \in \mathbb{R}^{1}: t \geqq 0\right\}$ characterized by

$$
\left.\begin{array}{lll}
0 \leqq f(t) \leqq 1, & f(0)=1, & f^{\prime}(0)=-1 \\
0 \leqq g(t) \leqq 1, & g(0)=1, & g^{\prime}(0)=-1
\end{array}\right\}
$$

Notice that $f(t A)^{\alpha} \stackrel{s}{\longrightarrow} I$ and $g(t B)^{\alpha} \stackrel{s}{\longrightarrow} I$ as $t \rightarrow+0$ for any $\alpha \geqq 0$. At the beginning we assume that

$$
0<f(t), \quad t \in \mathbb{R}_{+}^{1},
$$

and that

$$
\varphi(t)=\frac{1}{t}\left(\frac{1}{f(t)}-1\right) \text { and } \psi(t)=\frac{1}{t}(1-g(t))
$$

are monotonously nonincreasing functions . 
Condition (3.2) is necessary in order to give a correct statement of condition (3.3). The condition (3.3) itself has firstly been used by Kato in [6]. The conditions are satisfied for $f(t)=(1+k t)^{-k}, 0<k \leqq 1$, and $g(t)=e^{-t}$, for example.

In accordance with Kato [6] we define the family $\{M(t)\}_{t>0}$,

$$
M(t)=\frac{1}{t}\left[f(t A)^{-1}-g(t B)\right],
$$

of non-negative self-adjoint and, in general, unbounded operators. Since (3.1) and (3.2), the operators $M(t)$ are well-defined on $\mathscr{D}(M(t))=\mathscr{R}(f(t A)), t>0$.

Furthermore, we assume that

$$
f(t A) \in \mathscr{C}_{p}(\mathscr{H}), t>0,1 \leqq p<+\infty .
$$

Lemma 3.1. If the conditions (3.1)-(3.3) and (3.5) are satisfied, then

$$
(\lambda+M(t))^{-1} \in \mathscr{C}_{p}(\mathscr{H})
$$

for $\lambda>0$ and $t>0$.

Proof. On account of the identity

$$
(\lambda+M(t))^{-1}=t f(t A)^{1 / 2}\left\{I+f(t A)^{1 / 2}(\lambda t-g(t B)) f(t A)^{1 / 2}\right\}^{-1} f(t A)^{1 / 2},
$$

$\lambda, t>0$, the result follows if one proves that the operator in the curved brackets is boundedly invertible for $\lambda>0$ and $t>0$. For $\lambda t \geqq 1$ we get

$$
I+f(t A)^{1 / 2}(\lambda t-g(t B)) f(t A)^{1 / 2} \geqq I .
$$

For $0<\lambda t<1$ we get the inequality

$$
I+f(t A)^{1 / 2}(\lambda t-g(t B)) f(t A)^{1 / 2} \geqq \lambda t I .
$$

Lemma 3.2. Let $A$ and $B$ be two non-negative self-adjoint operators defined on the separable Hilbert $\mathscr{H}$ and let $f$ and $g$ be two Borel functions obeying (3.1)-(3.3). Let $F(t)=g(t B)^{1 / 2} f(t A) g(t B)^{1 / 2}, t \in \mathbb{R}_{+}^{1}$. If (3.5) is satisfied, then $e^{-t C} \in \mathscr{C}_{2 p}\left(\mathscr{H}^{\prime}\right)$ for $t>0$ and

$$
\|\cdot\|_{2 p^{-}} \lim _{n \rightarrow \infty} F\left(\frac{t}{n+1}\right)^{n}=e^{-t C} \oplus 0
$$

uniformly in $t \in[\eta, \tau], 0<\eta<\tau<+\infty$, where $C=A \dot{+} B$.

Proof. Let us introduce the operator-valued function $S(t)=t^{-1}[I-F(t)] \geqq 0, t>0$. Fixing $t>0$ in the sequel and setting $t^{\prime}=t / n$ and $\lambda=1 / t$ we get

$$
0 \leqq F\left(t^{\prime}\right)^{2 n} \leqq\left(I+\frac{1}{n \lambda} S\left(t^{\prime}\right)\right)^{-2 n} \leqq \lambda^{2}\left(\lambda+S\left(t^{\prime}\right)\right)^{-2}
$$

which implies $F\left(t^{\prime}\right)^{n} \ll \lambda\left(\lambda+S\left(t^{\prime}\right)\right)^{-1}$. Hence

$$
F\left(t^{\prime}\right)^{n+1} \ll \lambda\left(\lambda+S\left(t^{\prime}\right)\right)^{-1} F\left(t^{\prime}\right) .
$$


On the other hand, starting from the identity (3.7) one gets

$$
\begin{gathered}
g(t B)^{1 / 2}(\lambda+M(t))^{-1} g(t B)^{1 / 2}=(\lambda+S(t))^{-1} F(t) \\
+\lambda(\lambda+S(t))^{-1} g(t B)^{1 / 2}[I-f(t A)](\lambda+M(t))^{-1} g(t B)^{1 / 2},
\end{gathered}
$$

$\lambda, t>0$. Setting $t=t^{\prime}$ and $\lambda=1 / t$ as well as using $\left\|g\left(t^{\prime} B\right)^{1 / 2}\right\| \leqq 1,\left\|\lambda\left(\lambda+S\left(t^{\prime}\right)\right)^{-1}\right\| \leqq 1$ and $\|I-f(t A)\| \leqq 1$ we find

$$
\left\|\left(\lambda+S\left(t^{\prime}\right)\right)^{-1} F\left(t^{\prime}\right) u\right\| \leqq 2\left\|\left(\lambda+M\left(t^{\prime}\right)\right)^{-1} g\left(t^{\prime} B\right)^{1 / 2} u\right\|,
$$

$u \in \mathscr{H}$. Hence $\left(\lambda+S\left(t^{\prime}\right)\right)^{-1} F\left(t^{\prime}\right) \ll 2\left(\lambda+M\left(t^{\prime}\right)\right)^{-1} g\left(t^{\prime} B\right)^{1 / 2}$. But taking into account (3.9) we obtain

$$
F\left(t^{\prime}\right)^{n+1} \ll 2 \lambda\left(\lambda+M\left(t^{\prime}\right)\right)^{-1} g\left(t^{\prime} B\right)^{1 / 2} .
$$

By monotonicity (cf. [6]) we have $\left(\lambda+M\left(t^{\prime}\right)\right)^{-1} \leqq\left(\frac{1}{\tau}+M(\tau)\right)^{-1}, t \in\left[\frac{\eta}{3}, \tau\right]$. Thus

$$
F\left(\frac{t}{n}\right)^{n+1} \ll 2 \sqrt{\frac{3}{\eta}}\left(\frac{1}{\tau}+M(\tau)\right)^{-1 / 2} g\left(\frac{t}{n} B\right)^{1 / 2}
$$

$t \in\left[\frac{\eta}{3}, \tau\right]$. Substituting $t$ by $\frac{n}{n+2} t$ and then $n$ by $n-1$ we immediately obtain

$$
F\left(\frac{t}{n+1}\right)^{n} \ll 2 \sqrt{\frac{3}{\eta}}\left(\frac{1}{\tau}+M(\tau)\right)^{-1 / 2} g\left(\frac{t}{n+1} B\right)^{1 / 2},
$$

$t \in[\eta, \tau]$. Here $2 \sqrt{\frac{3}{\eta}}\left(\frac{1}{\tau}+M(\tau)\right)^{-1 / 2} \in \mathscr{C}_{2 p}(\mathscr{H}) \quad$ by $\quad$ (3.6). Since $s-\lim _{n \rightarrow \infty} F\left(\frac{t}{n+1}\right)^{n+1}=e^{-t C} \oplus 0$ (see [6,7]), and $s$ - $\lim _{n \rightarrow \infty} F\left(\frac{t}{n+1}\right)=I$ we obtain $s-\lim _{n \rightarrow \infty} F\left(\frac{t}{n+1}\right)^{n}=e^{-t c} \oplus 0, \quad t>0 . \quad$ Setting $\quad K_{n}(t)=F\left(\frac{t}{n+1}\right)^{n}$, $L=2 \sqrt{\frac{3}{\eta}}\left(\frac{1}{\tau}+M(\tau)\right)^{-1 / 2}, J_{n}(t)=g\left(\frac{t}{n+1} B\right)^{1 / 2}$ and applying Proposition 2.1 we get $e^{-t C} \in \mathscr{C}_{2 p}\left(\mathscr{H}^{\prime}\right)$ and $\|\cdot\|_{2 p}-\lim _{n \rightarrow \infty} F\left(\frac{t}{n+1}\right)^{n}=e^{-t C} \oplus 0$ for every $t>0$.

In order to show the uniformity of (3.8), we note that under our assumptions we have only to check that $s-\lim _{n \rightarrow \infty} f\left(\frac{t}{n+1} A\right)=I$ and

$$
s-\lim _{n \rightarrow \infty} g\left(\frac{t}{n+1} B\right)=I
$$


uniformly in $t \in[0, \tau]$. For instance, denoting by $E_{B}(\cdot)$ the spectral measure of $B$ we get the representation

$$
\begin{gathered}
\left\|\left(I-g\left(\frac{t}{n+1} B\right)\right) u\right\|^{2}=\int_{-\infty}^{+\infty}\left|1-g\left(\frac{t}{n+1} \lambda\right)\right|^{2} d\left(E_{B}(\lambda) u, u\right) \\
=\frac{t^{2}}{(n+1)^{2}} \int_{-\infty}^{+\infty}\left|\frac{1-g\left(\frac{t}{n+1} \lambda\right)}{\frac{t}{n+1} \lambda}\right|^{2} \lambda^{2} d\left(E_{B}(\lambda) u, u\right),
\end{gathered}
$$

$u \in \mathscr{H}$. Assuming $u \in \mathscr{D}(B)$ and taking into account (3.1) we obtain the estimate

$$
\left\|\left(I-g\left(\frac{t}{n+1} B\right)\right) u\right\| \leqq \text { const. } \frac{t}{n+1}\|B u\| \leqq \text { const. } \frac{\tau}{n+1}\|B u\|,
$$

$t \in[0, \tau]$, which yields the uniformity of $\lim _{n \rightarrow \infty} g\left(\frac{t}{n+1} A\right) u=u$ for every $u \in \mathscr{D}(B)$. Since $\mathscr{D}(B)$ is dense in $\mathscr{H}$ and $I-g\left(\frac{t}{n+1} B\right)$ is uniformly bounded we obtain (3.10).

The estimate $\left\|\left(I-g\left(\frac{t}{n+1} B\right)^{1 / 2}\right) u\right\| \leqq\left(I-g\left(\frac{t}{n+1} B\right)\right) u \|$ yields the uniformity of $s-\lim _{n \rightarrow \infty} g\left(\frac{t}{n+1} B\right)^{1 / 2}=I$ in $t \in[0, \tau]$. Since $s-\lim _{n \rightarrow \infty} f\left(\frac{t}{n+1} B\right)=I$ and $s-\lim _{n \rightarrow \infty} g\left(\frac{t}{n+1} B\right)^{1 / 2}=I$ uniformly in $t \in[0, \tau]$, we get $s-\lim _{n \rightarrow \infty} F\left(\frac{t}{n+1}\right)=I$ uniformly in $t \in[0, \tau]$. Therefore, by the uniformity of $s-\lim _{n \rightarrow \infty} F\left(\frac{t}{n+1}\right)^{n+1}=e^{-t C} \oplus 0$ in $t \in[\eta, \tau]$ (cf. [6]) we find the same for $s-\lim _{n \rightarrow \infty} F\left(\frac{t}{n+1}\right)^{n}=e^{-t c} \oplus 0$. Taking into account $\left\|g\left(\frac{t}{n+1} B\right)\right\| \leqq 1, t \in \mathbb{R}_{+}^{1}$, and applying Corollary 2.2 we complete the proof.

Let a $\{f, g\}$ be a pair of Borel functions obeying only the condition (3.1). With such a pair we can associate a pair $\left\{f_{0}, g_{0}\right\}$ of Borel functions such that the conditions (3.1)-(3.3) and, additionally, $0 \leqq f(t) \leqq f_{0}(t)$ and $0 \leqq g(t) \leqq g\left(t_{0}\right), t \in \mathbb{R}_{+}^{1}$, are satisfied. To prove this we introduce the pair $\left\{\varphi_{0}, \psi_{0}\right\}$ defined as follows:

$$
\begin{aligned}
& 0 \leqq \varphi_{0}(t)=\inf _{0<s \leqq t} \varphi(s)=\inf _{0<s \leqq t} s^{-1}\left(\frac{1}{f(s)}-1\right) \leqq 1, \\
& 0 \leqq \psi_{0}(t)=\inf _{0<s \leqq t} \psi(s)=\inf _{0<s \leqq t} s^{-1}(1-g(s)) \leqq 1
\end{aligned}
$$

where we agree to set $\frac{1}{f(s)}=+\infty$ if $f(s)=0$. Defining $f_{0}(t)$ by

$$
f_{0}(t)= \begin{cases}1, & t=0 \\ \left(1+t \varphi_{0}(t)\right)^{-1}, & t>0\end{cases}
$$


we get $0<f_{0}(t) \leqq 1$ (condition (3.2) for $f_{0}$ ) and

$$
0 \leqq f(t) \leqq f_{0}(t) \leqq 1 \text {. }
$$

Since $\lim _{t \rightarrow+0} f(t)=1$, we find $\lim _{t \rightarrow+0} f_{0}(t)=1$. Moreover, we have

$$
1=\lim _{t \rightarrow+0} \varphi(t)=\lim _{t \rightarrow+0} \inf \varphi(t)=\lim _{t \rightarrow+0} \varphi_{0}(t)=1 .
$$

Thus, using $\lim _{t \rightarrow+0} f_{0}(t)=1$ we find

$$
\lim _{t \rightarrow+0} \frac{f_{0}(t)-1}{t}=-\lim _{t \rightarrow+0} \frac{\varphi_{0}(t)}{1+t \varphi_{0}(t)}=-\lim _{t \rightarrow+0} \varphi_{0}(t) f_{0}(t)=-1 \text {. }
$$

Consequently, $f_{0}$ obeys (3.1). Since $t^{-1}\left(\frac{1}{f_{0}(t)}-1\right)=\varphi_{0}(t)$ and $\varphi_{0}(t)$ is monotonously nonicnreasing by construction, the condition (3.3) is satisfied. Let

$$
g_{0}(t)= \begin{cases}1, & t=0 \\ 1-t \psi(t), & t>0\end{cases}
$$

Since $\psi_{0}(t) \leqq \psi(t), t>0$, we get $0 \leqq g(t)=1-t \psi(t) \leqq 1-t \psi_{0}(t)=g_{0}(t), t>0$. Furthermore, the representation

$$
0 \leqq \psi_{0}(t)=t^{-1}\left(1-g_{0}(t)\right)
$$

implies $0 \leqq 1-g_{0}(t)$ or $g_{0}(t) \leqq 1, t>0$. Thus, summing up we obtain $0 \leqq g(t) \leqq g_{0}(t) \leqq 1$. Taking into account

$$
1=\lim _{t \rightarrow+0} \psi(t)=\lim _{t \rightarrow+0} \inf \psi(t)=\lim _{t \rightarrow+0} \psi_{0}(t)=1
$$

we find $\lim _{t \rightarrow+0} \frac{g_{0}(t)-1}{t}=-\lim _{t \rightarrow+0} \psi_{0}(t)=-1$. Hence, $g_{0}$ obeys (3.1). Since $t^{-1}\left(1-g_{0}(t)\right)=\psi_{0}(t)$ is monotonously nonincreasing by construction, the condition (3.3) is valid.

Therefore, starting with the functions $f$ and $g$ obeying (3.1) we have constructed an associated pair $\left\{f_{0}, g_{0}\right\}$ of Borel functions satisfying (3.1)-(3.3).

In order to apply the previous considerations, we replace the condition (3.5) by

$$
f_{0}(t A) \in \mathscr{C}_{p}(\mathscr{H}), t>0,1 \leqq p<+\infty .
$$

For example, let $f(t)=e^{-t}$. It is easy to check that in this case $f_{0}(t)=(1+t)^{-1}$. Hence, (3.12) means that $(I+t A)^{-1} \in \mathscr{C}_{p}(\mathscr{H}), t>0,1 \leqq p<+\infty$.

Lemma 3.3. Let $F_{0}(t)=\left(g_{0}(t B)\right)^{1 / 2} f_{0}(t A)\left(g_{0}(t B)\right)^{1 / 2}$. If the condition (3.12) is satisfied, then $f(t A) \in \mathscr{C}_{p}(\mathscr{H}), t>0,1 \leqq p<+\infty$, and

for $m \geqq 1$.

$$
\left\|F(t)^{m}\right\|_{p} \leqq\left\|F_{0}(t)^{m}\right\|_{p}, \quad t>0,
$$

Proof. The first assertion follows from (3.11) and (3.12). Now let $\hat{F}(t)$ $=(g(t B))^{1 / 2} f_{0}(t A)(g(t B))^{1 / 2}$. Since $F(t) \leqq \hat{F}(t)$ we get [13]

$$
\left\|F(t)^{m}\right\|_{p}^{p}=\operatorname{Tr}\left\{F(t)^{m p}\right\} \leqq \operatorname{Tr}\left\{\hat{F}(t)^{m p}\right\}=\left\|\hat{F}(t)^{m}\right\|_{p}^{p}
$$


Let $\check{F}(t)=\left(f_{0}(t A)\right)^{1 / 2} g(t B)\left(f_{0}(t A)\right)^{1 / 2}$ and $\check{F}_{0}(t)=\left(f_{0}(t A)\right)^{1 / 2} g_{0}(t B)\left(f_{0}(t A)\right)^{1 / 2}$. Obviously, one has

$$
\left\|\widehat{F}(t)^{m}\right\|_{p}^{p}=\operatorname{Tr}\left\{\widehat{F}(t)^{m p}\right\}=\operatorname{Tr}\left\{\check{F}(t)^{m p}\right\}=\left\|\check{F}(t)^{m}\right\|_{p}^{p}
$$

and, analogously,

$$
\left\|\check{F}_{0}(t)^{m}\right\|_{p}^{p}=\left\|F_{0}(t)^{m}\right\|_{p}^{p} .
$$

On account of (3.14), (3.15), (3.16) and $\check{F}(t) \leqq \check{F}_{0}(t)$ we find

$$
\left\|F(t)^{m}\right\|_{p} \leqq\left\|\check{F}(t)^{m}\right\|_{p} \leqq\left\|\check{F}_{0}(t)^{m}\right\|_{p}=\left\|F_{0}(t)^{m}\right\|_{p}
$$

which proves (3.13).

Theorem 3.4. Let $A$ and $B$ be two non-negative self-adjoint operators defined in the separable Hilbert space $\mathscr{H}$ and let $f$ and $g$ be two Borel functions obeying (3.1). If (3.12) is satisfied, then $e^{-t C} \in \mathscr{C}_{1}\left(\mathscr{H}^{\prime}\right), t>0$, and

$$
\|\cdot\|_{1}-\lim _{\substack{n \rightarrow \infty \\(n \geqq p)}}\left(f\left(\frac{t}{n} A\right) g\left(\frac{t}{n} B\right)\right)^{n}=e^{-t C} \oplus 0
$$

holds uniformly in $t \in[\eta, \tau], 0<\eta<\tau<+\infty$.

Proof. By Lemma 3.2 we have $e^{-(t / 2 p) C} \in \mathscr{C}_{2 p}\left(\mathscr{H}^{\prime}\right), t>0$. Since $\left(e^{-(t / 2 p) C}\right)^{2 p}=e^{-t C}$ we get $e^{-t C} \in \mathscr{C}_{1}\left(\mathscr{H}^{\prime}\right), t>0$.

Next we show that

$$
\|\cdot\|_{k}-\lim _{n \rightarrow \infty}\left(f\left(\frac{t}{n} A\right) g\left(\frac{t}{n} B\right)\right)^{n}=e^{-t C} \oplus 0
$$

uniformly in $t \in\left[\frac{\eta^{\prime}}{k}, \frac{\tau}{k}\right]$, where $\eta^{\prime}$ is an arbitrary real number obeying $0<\eta^{\prime}<\eta$ and $k$ is a natural number greater than $2 p$, i.e. $2 p \leqq k$. Using Lemma 3.3 we find

$$
\begin{aligned}
& \left\|\left(f\left(\frac{t}{n} A\right) g\left(\frac{t}{n} B\right)\right)^{n}\right\|_{2 p}=\left\|f\left(\frac{t}{n} A\right) g\left(\frac{t}{n} B\right)^{1 / 2} F\left(\frac{t}{n}\right)^{n-1} g\left(\frac{t}{n} B\right)^{1 / 2}\right\|_{2 p} \\
& \leqq\left\|F\left(\frac{t}{n}\right)^{n-1}\right\|_{2 p} \leqq\left\|F_{0}\left(\frac{t}{n}\right)^{n-1}\right\|_{2 p} \text {. }
\end{aligned}
$$

Furthermore, we note that $s-\lim _{n \rightarrow \infty}\left(f\left(\frac{t}{n} A\right) g\left(\frac{t}{n} B\right)\right)^{n}=e^{-t C} \oplus 0 \quad$ and $\|\cdot\|_{2 p}-\lim _{n \rightarrow \infty} F_{0}\left(\frac{t}{n}\right)^{n-1}=e^{-t C} \oplus 0$ uniformly in $t \in\left[\frac{\eta^{\prime}}{k}, \frac{\tau}{k}\right]$ by [7] and Lemma 3.2, respectively. Setting $K_{n}(t)=\left(f\left(\frac{t}{n} A\right) g\left(\frac{t}{n} B\right)\right)^{n}, \hat{K}_{n}(t)=F_{0}\left(\frac{t}{n}\right)^{n-1}, L=e^{-\eta C} \oplus 0$, taking into account (3.19) and applying Corollary 2.4 we prove (3.18).

Since the convergence of (3.18) is uniform we obtain

$$
\|\cdot\|_{k}-\lim _{n \rightarrow \infty}\left(f\left(\frac{t}{k n+l} A\right) g\left(\frac{t}{k n+l} B\right)\right)^{n}=e^{-(t / k) c} \oplus 0
$$


uniformly in $t \in[\eta, \tau]$ and in $l=0,1,2, \ldots, k-1$. By the estimate

$$
\begin{aligned}
& \left\|\left(f\left(\frac{t}{k n+l} A\right) g\left(\frac{t}{k n+l} B\right)\right)^{k n}-\left\{e^{-(t / k) c} \oplus 0\right\}^{k}\right\|_{1} \\
& \quad \leqq \sum_{m=0}^{k-1}\left\|\left(f\left(\frac{t}{k n+l} A\right) g\left(\frac{t}{k n+l} B\right)\right)^{n}\right\|_{k}^{m} \|\left(f\left(\frac{t}{k n+l} A\right) g\left(\frac{t}{k n+l} B\right)\right)^{n} \\
& \quad-\left\{e^{-(t / k) c} \oplus 0\right\}\left\|_{k}\right\| e^{-(t / k) C} \oplus 0 \|_{k}^{k-m-1},
\end{aligned}
$$

$t \in[\eta, \tau]$, and (3.20) we get

$$
\|\cdot\|_{1}-\lim _{n \rightarrow \infty}\left(f\left(\frac{t}{k n+l} A\right) g\left(\frac{t}{k n+l} B\right)\right)^{k n}=e^{-t C} \oplus 0
$$

uniformly in $t \in[\eta, \tau]$ and in $l=0,1,2, \ldots, k-1$. By considerations similar to those of Lemma 3.2 we find that $s-\lim _{n \rightarrow \infty} f\left(\frac{t}{k n+l} A\right)=I$ and $s-\lim _{n \rightarrow \infty} g\left(\frac{t}{k n+l} B\right)=I$ uniformly in $t \in[0, \tau]$ and in $l=0,1,2, \ldots, k-1$. Thus, it is easy to see that $s-\lim _{n \rightarrow \infty}\left(f\left(\frac{t}{k n+l} A\right) g\left(\frac{t}{k n+l} B\right)\right)^{l}=I$ uniformly in $t \in[0, \tau]$ and in $l=0,1,2, \ldots, k-1$. Hence we obtain that

$$
\|\cdot\|_{1}-\lim _{n \rightarrow \infty}\left(f\left(\frac{t}{k n+l} A\right) g\left(\frac{t}{k n+l} B\right)\right)^{k n+l}=e^{-t C} \oplus 0
$$

uniformly in $t \in[\eta, \tau]$ and $l=0,1,2, \ldots, k-1$. But the last assertion coincides with (3.17).

\section{Application and Conclusion}

As an application of the above results for the Gibbs semigroups, we mention continuous systems of the QSM in a finite volume.

Let us consider an $N$-particle system enclosed in a box $\Lambda \subset \mathbb{R}^{v}$ which is a bounded, open, connected subset of the $v$-dimensional Euclidean space with a smooth boundary $\partial \Lambda$. Hence, the appropriate Hilbert space is $\mathscr{H}=L^{2}\left(\Lambda^{N}\right)$. In our discussion, the statistics of particles is not important, therefore, we ignore the symmetry of the wave function $\psi \in \mathscr{H}$. The kinetic-energy operator $T_{\sigma}$ for the particles of the mass $m$ is a self-adjoint extension of the sum

$$
T_{N}=\sum_{j=1}^{N}\left(-\frac{1}{2 m} \Delta_{j}\right), \quad \Delta_{j}=\sum_{\alpha=1}^{v} \partial_{j, \alpha}^{2},
$$

with the domain $\mathscr{D}\left(T_{N}\right)=C_{0}^{\infty}\left(\Lambda^{N}\right)$. The domain $\mathscr{D}\left(T_{\sigma}\right)$ is specified by a boundary condition $\sigma \in C(\partial \Lambda)$. Then, one can check that $T_{\sigma}$ is a $p$-generator for the selfadjoint Gibbs semigroup $G_{T_{\sigma}}(t)[12]$, i.e., for $\frac{N v}{2} \leqq p<+\infty$ we have

$$
\left(z-T_{6}\right)^{-1} \in \mathscr{C}_{p}(\mathscr{H}), \quad z \in \mathbb{C} \backslash \mathbb{R}_{+}^{1} .
$$


The stable particle interaction $U_{N} \geqq-N u I$ is a self-adjoint multiplication operator with a real-valued measurable function defined on the domain $\mathscr{D}\left(U_{N}\right)$ $=\left\{\psi \in \mathscr{H}: U_{N} \psi \in \mathscr{H}\right\}$, see e.g. [17].

Therefore, we have to generalize our results a bit to include the semiboundedness of the operator $U_{N}$. Moreover, to describe the short-distance behaviour of the two-body interaction, a hard-core potential is frequently used [17]. Then, the original Hilbert space of the wave functions $\mathscr{H}=L^{2}\left(\Lambda^{N}\right)$ should be reduced to $\mathscr{H}^{\prime}=\Pi \mathscr{H}=L^{2}\left(\Lambda^{N} \backslash S_{N}\right)$, where $S_{N}$ is a region forbidden by hard-cores.

Theorem 4.1. Let $T_{\sigma} \geqq 0$ and $U_{N} \geqq-N u I$ be self-adjoint operators defined in a separable Hilbert space $\mathscr{H}=L^{2}\left(\Lambda^{N}\right)$. Let $H=T_{\sigma} \dot{+} U_{N}$ and let $\Pi$ be the orthogonal projection from $\mathscr{H}$ onto $\mathscr{H}^{\prime}=L^{2}\left(\Lambda^{N} \backslash S_{N}\right)$ spanned by $Q=\mathscr{D}\left(T_{\sigma}^{1 / 2}\right) \cap \mathscr{D}\left(\left(U_{N}\right.\right.$ $\left.+N u I)^{1 / 2}\right)$. If (4.1) is satisfied, then

$$
\|\cdot\|_{1}-\lim _{n \rightarrow \infty}\left\{\exp \left(-\frac{\beta}{n} T_{\sigma}\right) \exp \left(-\frac{\beta}{n} U_{N}\right)\right\}^{n}=e^{-\beta H} \Pi
$$

uniformly in the inverse temperature $\beta>0$ varying in a compact interval bounded away from zero.

Proof. Let us introduce $A=T_{\sigma} \geqq 0$ and $B=U_{N}+N u I \geqq 0$. Then by (4.1) we get that the condition (3.12) is fulfilled. Applying Theorem 3.4 we get

$$
\|\cdot\|_{1}-\lim _{n \rightarrow \infty}\left(e^{-(\beta / n) T_{\sigma}} e^{-(\beta / n)\left(U_{N}+N u I\right)}\right)^{n}=e^{-\beta C} \Pi
$$

uniformly in $\beta>0$ on any compact interval bounded away from zero, where $C=A \dot{+} B=H+N u I$. Cancelling both parts of $(4.3)$ by $\exp (-\beta u N)$ one gets (4.2).

Remarks 4.2. Our results are not applicable to interactions $U$ which are not semibounded from below (e.g. for Coulomb systems) in spite of the semiboundedness of the operator $H$.

Acknowledgement. The authors wish to thank the referee for his hints which have allowed them to essentially simplify the proofs of their earlier work.

\section{References}

1. Trotter, H.F.: On the products of semigroups of operators. Proc. Am. Math. Soc. 10, 545-551 (1959)

2. Chernoff, P.R.: Product formulas, nonlinear semigroups, and addition of unbounded operators. Mem. Am. Math. Soc. 140, 1-121 (1974)

3. Faris, W.G.: The product formula for semigroups defined by Friedrichs extension. Pacific J. Math. 22, 47-70 (1967)

4. Simon, B.: Quantum Mechanics for Hamiltonians defined as quadratic forms. Princeton, N.J.: Princeton University Press 1971

5. Kato, T.: Perturbation theory of linear operators. (2nd ed.) Berlin, Heidelberg, New York: Springer 1976

6. Kato, T.: On the Trotter-Lie product formula. Proc. Jpn. Acad. 50, 694-698 (1974)

7. Kato, T.: Trotter's product formula for an arbitrary pair of self-adjoint contraction semigroups. Topics in Funct. Anal., Ad. Math. Suppl. Studies, Vol. 3, 185-195. Gohberg, I., Kac, M. (eds.). New York: Academic Press 1978 
8. Lapidus, M.L.: The problem of the Trotter-Lie formula for unitary groups of operators. Seminaire Choquet, Publ. Math. Université Pierre-et-Marie-Curie 46, 1701-1745 (1982)

9. Lapidus, M.L.: Product formula for imaginary resolvents with application to a modified Feynman integral. J. Funct. Anal. 63, 261-275 (1985)

10. Zagrebnov, V.A.: The Trotter-Lie product formula for Gibbs semigroups. J. Math. Phys. 29, 888-891 (1988)

11. Zagrebnov, V.A.: On the families of Gibbs semigroups. Commun. Math. Phys. 76, 269-276 (1980)

12. Zagrebnov, V.A.: Perturbations of Gibbs semigroups. Commun. Math. Phys. 120, 653-664 (1989)

13. Simon, B.: Trace ideals and their applications. London Math. Soc. Lecture Notes Ser., Vol. 35. Cambridge: Cambridge University Press 1979

14. Grümm, H.R.: Two theorems about $\mathscr{C}_{p}$. Rep. Math. Phys. 4, 211-215 (1973)

15. Zagrebnov, V.A.: On singular potential interactions in quantum statistical mechanics. Trans. Moscow Math. Soc. 41, 101-120 (1980)

16. Bratteli, O., Robinson, D.W.: Operator algebras and quantum statistical mechanics, Vol. II. Berlin, Heidelberg, New York: Springer 1981

17. Ruelle, D.: Statistical mechanics. Rigorous results. New York: Benjamin 1969

Communicated by H. Araki

Received April 19, 1989; in revised form December 7, 1989 